Short communication

\title{
ANTIBIOTIC SUSCEPTIBILITY, SEROTYPING AND PATHOGENICITY EVALUATION OF AVIAN ESCHERICHIA COLI ISOLATED FROM BROILERS IN NORTHERN IRAN
}

\author{
S. SEIFI ${ }^{1}$, R. KHOSHBAKHT ${ }^{1} \&$ A. R. ATABAK ${ }^{2}$ \\ ${ }^{1}$ Faculty of Veterinary Medicine, Amol University of Special Modern \\ Technologies, Amol, Iran; ${ }^{2}$ Amol Joojeh Company, Amol, Iran
}

\section{Summary}

Seifi, S., R. Khoshbakht \& A. R. Atabak, 2015. Antibiotic susceptibility, serotyping and pathogenicity evaluation of avian Escherichia coli isolated from broilers in northern Iran. Bulg. J. Vet. Med., 18, No 2, 173-179.

The aim of this study was to determine the main serotypes of avian-associated $E$. coli and their susceptibilities to common antimicrobial agents used in Iranian poultry industry. Furthermore the invasion characteristics of the poultry-associated isolates were evaluated. Eighty Escherichia coli (E. coli) strains were isolated from 63 broiler farms with colibacillosis clinical signs in Mazandaran province, northern of Iran. Antibacterial susceptibility testing of the isolates with ten selected antibiotics was conducted according to the standard methods and avian pathogen serotypes, O1, O2, O18 and O78:K80, were determined using the specific antisera. In addition, invasion properties of the isolates were examined based on Congo red dye agar test. According to the results, $73.75 \%, 71.25 \%$ and $65 \%$ of isolates were resistant to oxytetracycline, tetracycline and erythromycin, respectively, while resistance rates against enrofloxacin, florfenicol and norfloxacin were $7.5 \%, 7.5 \%$ and $2.5 \%$ respectively. The $\mathrm{O} 78$ and $\mathrm{O} 1$ were the predominant serotypes among $E$. coli isolates. Nine resistance patterns were observed in the $E$. coli isolates with predominant patterns being distributed widely across broilers indicating a striking diversity of resistance patterns in the areas. Cross-resistance between animal and human antimicrobial agents may be possible, thus, their proper usage in veterinary medicine is necessary.

Key words: broilers, Escherichia coli, Iran, multi-drug resistance, serotyping

Colibacillosis in chickens refers to any localised or systemic infection caused entirely or partly by Escherichia coli (Barnes et al., 2003), enteric Gramnegative, flagellated bacterium which is part of the normal flora in the digestive tract of chickens (Nakamura et al., 1992). Although the microorganism has low pathogenicity for chickens, pathogenic avian strains of E. coli (APEC) are identified, which belong to a small range of serotypes including O78:K80, O1:K1 and 
Antibiotic susceptibility, serotyping and pathogenicity evaluation of avian Escherichia coli...

O2:K1 (Barnes et al., 2003). Several pathological signs due to infection with an E. coli strain can be distinguished in poultry: septicaemia, granuloma, inflammation of air sacs, cellulitis, swollen head syndrome, peritonitis, salpingitis, osteomyelitis, panophthalmitis and omphalitis/yolk sac infection. In broilers, colibacillosis mainly results in respiratory infections (airsacculitis) and peritonitis/pericarditis (Pourbakhsh et al., 1997). Colibacillosis is mainly treated with antibiotics, but the use of these drugs is costly and the period in which broilers can be treated is limited because of the withdrawal time. Also, significant increase in appearance of antibiotic-resistant strains of $E$. coli isolated from poultry has complicated the problem (Khoshkhoo \& Peighambari, 2005; Johnson et al., 2007). Serotyping of the antigens is a very useful method for detecting pathogenic $E$. coli strains in clinical specimens, foods, and environmental samples and for understanding the epidemiology of the pathogen (Wang et al., 2010). In this study, colibacillosis related E. coli strains isolated from poultry farms were analysed to determine the main serotypes of avian-associated E. coli and their susceptibilities to common antimicrobial agents used in Iranian poultry industry. Furthermore, the invasion characteristics of the poultry-associated isolates were evaluated.

The study was conducted in Mazandaran province (Northern Iran) during May and December 2012. Liver samples were collected aseptically from chickens with severe clinically signs after necropsy from 63 colibacillosis-affected broiler farms (20 liver samples per flock in four batches) and were placed into sterile Whirl-pak bags. Clinical signs observed in depressed birds were sneezing and coughing, and the most significant gross lesion was airsacculitis. No antibiotic treatment was used prior to sampling time and the specimens were submitted to the laboratory on ice pieces in less than $6 \mathrm{~h}$.

Homogenised samples were inoculated onto MacConkey's agar (Merck, Germany) plates and incubated at $37{ }^{\circ} \mathrm{C}$ for 24 h. Suspected Escherichia coli colonies were subsequently inoculated on Eosin Methylene Blue (EMB) agar (HiMedia, India) and incubated as above. Isolates were Gram-stained, tested for catalase and oxidase, sulfide-indole-motility (SIM), methyl red, Voges-Proskauer, citrate (IMVIC), triple sugar iron (TSI), and then stored on nutrient agar until used for antimicrobial susceptibility tests. Antimicrobial drug susceptibility was determined by a disc-diffusion method on MuellerHinton (MH) agar plates (Merck, Germany), according to the antibiogram standard methods (Bauer et al., 1966). The following antibiotic discs were applied: ampicillin $(10 \mu \mathrm{g})$, amoxicillin $(25 \mu \mathrm{g})$, co-amoxiclav $(20 \mu \mathrm{g})$, tetracycline $(30$ $\mu \mathrm{g})$, oxytetracycline $(30 \mu \mathrm{g})$, erythromycin $(15 \mu \mathrm{g})$, florfenicol $(30 \mu \mathrm{g})$, flumequine (30 $\mu \mathrm{g})$, enrofloxacin $(5 \mu \mathrm{g})$, and norfloxacin $(10 \mu \mathrm{g})$. All antibiotic discs obtained from Padtan Teb, Iran.

Avian pathogenic strains were determined by agglutination test with specific antiserum raised against $\mathrm{O} 1, \mathrm{O} 2, \mathrm{O} 18$, and O78 antigens (Sifin, Bioconnections, UK) according to the protocol previously described (Allan et al., 1993). Isolates pathogenicity test was carried out based on Congo red dye agar test previously explained by Berkhoff \& Vinal (1986). Briefly, the colonies were streaked on Congo red agar (HiMedia, India) and incubated for $72 \mathrm{~h}$ at $25{ }^{\circ} \mathrm{C}$. The reaction was detected at 18, 24, 48 and $72 \mathrm{~h}$. Appearance of red colonies within $72 \mathrm{~h}$ was recorded as a positive reaction (invasive 
strains). Negative colonies did not bind the dye and remained white or grey even after $72 \mathrm{~h}$ and were declared negative.

A total of $80 \mathrm{E}$. coli isolates were recovered from 63 clinically affected broiler farms in Mazandaran province, Iran.

Regarding the individual antimicrobials, E. coli resistance was observed for all 10 antimicrobial agents. The isolates showed high levels of resistance $(>50 \%)$ to tetracycline $(71.25 \%)$, oxytetracycline $(73.75 \%)$, erythromycin $(65 \%)$, and ampicillin $(62.5 \%)$. The antimicrobials detected at low levels of resistance $(<10 \%)$ were enrofloxacin $(7.5 \%)$, norfloxacin $(2.5 \%)$ and florfenicol (7.5\%) (Table 1). Antimicrobial resistance levels of the broiler-originated $E$. coli isolates to the tetracycline group (71.25\%-73.75\%) dominated over other groups while their antimicrobial susceptibility level to the fluoroquinolone group was still high (resistance levels were less than $10 \%$ ). With antimicrobials from the $\beta$-lactam groups, levels of resistance of $E$. coli isolates were higher to the older (ampicillin and amoxicillin) than to newer antimicrobials (coamoxiclav). Each of the eighty E. coli isolates showed resistance to at least two antibiotics. Table 2 shows the distribution of isolates into different groups of resistance patterns and the percentage of occurrence in relation to total isolates.

Among the 80 E. coli strains $86.25 \%$ belonged to the four serogroups with 17 to $\mathrm{O} 1,14$ to $\mathrm{O} 2,8$ to $\mathrm{O} 18$ and 30 to $\mathrm{O} 78$ (Table 3 ). Out of the eighty E. coli isolates subjected to Congo red binding assay, $71(88.75 \%)$ isolates were positive and $9(11.25 \%)$ isolates were negative.

Antimicrobial resistance among bacteria isolated from food animals is a matter of concern to public health and animals as well. Investigations on $E$. coli isolates from poultry have shown increased resistance to antimicrobials (Khoshkhoo \& Peighambari, 2005; Li et al., 2010). Johnson et al. (2007) demonstrated a close similarity between resistant human and poultry E. coli isolates.

In this study, fifty $(62.5 \%)$ of all isolates were resistant to ampicillin. High percentages $(89.6 \%$ and $96.7 \%)$ of resistance to ampicillin were reported by Gundogan et al. (2006) and Khoshkhoo \& Peighambari (2005), respectively.

Table 1. Antibiotic resistance patterns of $80 \mathrm{E}$. coli isolated from broilers with colibacilosis symptoms

\begin{tabular}{lccc}
\hline Antibiotic & $\begin{array}{c}\text { Number (\%) } \\
\text { of sensitive isolates }\end{array}$ & $\begin{array}{c}\text { Number (\%) } \\
\text { of intermediate isolates }\end{array}$ & $\begin{array}{c}\text { Number (\%) } \\
\text { of resistant isolates }\end{array}$ \\
\hline A $(10 \mu \mathrm{g})$ & $18(22.50)$ & $12(15.00)$ & $50(62.50)$ \\
\hline AMX $(25 \mu \mathrm{g})$ & $46(57.50)$ & $8(10.00)$ & $26(32.50)$ \\
\hline AMC $(20 \mu \mathrm{g})$ & $59(73.75)$ & $7(8.75)$ & $14(17.50)$ \\
TE $(30 \mu \mathrm{g})$ & $3(3.75)$ & $10(12.50)$ & $57(71.25)$ \\
OTC $(30 \mu \mathrm{g})$ & $7(8.75)$ & $14(17.50)$ & $59(73.75)$ \\
E $(15 \mu \mathrm{g})$ & $14(17.50)$ & $14(17.50)$ & $52(65.00)$ \\
FF $(30 \mu \mathrm{g})$ & $58(72.50)$ & $16(20.00)$ & $6(7.50)$ \\
FM $(30 \mu \mathrm{g})$ & $33(41.25)$ & $8(10.00)$ & $39(48.75)$ \\
NFX $(5 \mu \mathrm{g})$ & $66(82.50)$ & $8(10.00)$ & $6(7.50)$ \\
NX $(10 \mu \mathrm{g})$ & $71(88.75)$ & $7(8.75)$ & $2(2.50)$ \\
\hline
\end{tabular}

A, ampicillin; AMX, amoxicillin; AMC, co-amoxiclav; TE, tetracycline; OTC, oxytetracycline; E, erythromycin; FF, florfenicol; FM, flumequine; NFX, enrofloxacin; NX, norfloxacin. 
Antibiotic susceptibility, serotyping and pathogenicity evaluation of avian Escherichia coli...

Table 2. Multiresistance patterns of $E$. coli isolates. The left column present the number of tested drugs against which the isolates are resistant, the right one - drug combinations. The number of isolates resistant against each combination is given in brackets.

\begin{tabular}{cl}
\hline Number of drugs & Drug combinations (number of isolates) \\
\hline 2 & OTC+TE (1) \\
& A+TE+OTC (2); \\
3 & A+OTC+AMX (1); \\
& TE+OTC+E (3) \\
& A+TE+OTC+E (10); \\
4 & TE+OTC+E+FM (2) \\
5 & A+TE+OTC+E+FM (12) \\
6 & A+TE+OTC+AMX+E+FM (11) \\
7 & AMC+A+TE+OTC+AMX+E+FM (5); \\
& AMC+A+TE+OTC+AMX+E+FM (1) \\
8 & AMC+A+TE+OTC+AMX+E+FM+FF(2); \\
9 & AMC+A+TE+OTC+AMX+E+FM+NFX (2) \\
10 & AMC+A+TE+OTC+AMX+E+FM+FF+NFX (2) \\
\hline
\end{tabular}

A, ampicillin; AMX, amoxicillin; AMC, co-amoxiclav; TE, tetracycline; OTC, oxytetracycline; E, erythromycin; FF, florfenicol; FM, flumequine; NFX, enrofloxacin; NX, norfloxacin.

Table 3. Prevalence of different serotypes of $E$. coli isolated from broilers with colibacilosis symptoms

\begin{tabular}{lcc}
\hline E. coli serotypes & Number of isolates (\%) & Number of invasive isolates (\%) \\
\hline O1 & $17(21.25)$ & $16(94.1)$ \\
O2 & $14(17.5)$ & $12(85.7)$ \\
O18 & $8(10)$ & $6(75)$ \\
O78: $($ K80) & $30(37.5)$ & $30(100)$ \\
Other serotypes & $11(13.75)$ & $7(63.6)$ \\
\hline Total & $80(100)$ & $71(88.7)$ \\
\hline
\end{tabular}

Two (2.5\%) out of 80 Escherichia coli isolates were resistant to norfloxacin, variable results were obtained by different authors. Khoshkhoo \& Peighambari (2005) and Alimehr et al. (1999) reported $52.7 \%$ and $20.4 \%$ resistance to norfloxacin among Escherichia coli isolates from broilers, respectively. On the other hand, $0 \%$ resistance to norfloxacin was mentioned by Ali Akond et al. (2009). Such differences may well be related to the source, frequency and type of $E$. coli isolates encountered in different geographical areas.

The results revealed that $6(7.5 \%)$ out a total of 80 Escherichia coli isolates were resistant to enrofloxacin. Similar results were obtained by different authors, 4\% and $9.3 \%$ of avian Escherichia coli were found resistant to enrofloxacin by Zakeri 
\& Kashefi (2012), and Hanson et al. (2002). In the current study, $6(7.5 \%)$ out of all isolates were resistant to florfenicol. Similar percentage of florfenicol resistant Escherichia coli (10\%) was recorded among avian Escherichia coli isolates by Gregova et al. (2012).

The results showed that the least sensitivity was related to tetracycline $(3.75 \%)$ and oxytetracycline $(8.75 \%)$. These results are in line with the results of Zakeri \& Kashefi (2012) and Hanson et al. (2002). High resistance to these antibiotics may be explained by the fact that both are frequently used by poultry industry in Iran.

Multi-drug resistance (MDR) appeared as a real problem as the majority of strains were resistant to at least two antibiotics. Increased MDR has been reported in $E$. coli isolates in many countries including Iran (Khoshkhoo \& Peighambari, 2005; Li et al., 2010). A similar finding on MDR of $E$. coli strains has been reported from other parts of the world (Khan et al., 2002; Guerra et al., 2003; Rahman et al., 2008). Antimicrobial susceptibility test is very important to choose efficient antimicrobial treatment against avian E. coli. Results showed that among MDR types, numbers of antimicrobial agents were different - between 2 to 12 . This is in line with the results of another study on poultry E. coli (Khoshkhoo \& Peighambari, 2005). Direct contacts and animal-origin foods can spread MDR bacterial isolates into human population (Soulsby, 2008). After colonisation of resistant bacteria in the human intestinal tract, the antibiotic resistance gene can be transferred to microflora or pathogenic bacteria. Shedding of resistant bacteria in the environment can infect animals and then come back to humans through the food chain (Hawkey, 2008).
Similar to previous studies (Cavalieri et al., 1984; Gross, 1994), present results showed that $078, \mathrm{O} 1$ and $\mathrm{O} 2$ were the dominant serotypes of $E$. coli in colibacilosis infected poultry. Also, Allan et al. (1993) showed that $61 \%$ of 44 isolates were typeable, with $\mathrm{O} 1,02$ and 078 being the most frequent serotypes.

The result of the Congo red binding assay indicated that the majority $(88.75 \%)$ of cases of colibacillosis produced Congo red positive colonies. In vitro Congo red pathogenicity test could be clearly correlated with invasion ability and pathogenicity. This finding is in agreement with a previous study (Berkhoff \& Vinal, 1986).

In conclusion, this study shows that multidrug resistant $E$. coli isolates are prevalent among broilers in Iran. It is recommended that the use of antimicrobial agents in food animals should follow prudent use guidelines to minimise the selection and spread of resistant bacteria. Also, utilisation of some antibiotics such as tetracycline, oxytetracycline and erythromycin in poultry farms should be revised. Moreover, it seems that antimicrobial resistance test former to therapy at the farm can be helpful for better antibiotic treatments.

\section{REFERENCES}

Ali Akond, M., S. M. R. Hassan, S. Alam \& M. Shirin, 2009. Antibiotic resistance of Escherichia coli isolated from poultry and poultry environment of Bangladesh. American Journal of Environmental Sciences, 5, 47-52.

Alimehr, M., G. Sadeghi-Hashjin, S. A. Pourbakhsh \& K. Norouzi, 1999. Isolation, Identification and in vitro susceptibility of avian Escherichia coli to selected fluoroquinolones. Archives of Razi Institute, 50, 77-82. 
Antibiotic susceptibility, serotyping and pathogenicity evaluation of avian Escherichia coli...

Allan, B. J., J. V. van den Hurk \& A. A. Potter, 1993. Characterization of Escherichia coli isolated from cases of avian colibacillosis. Canadian Journal of Veterinary Research, 57, 146-151.

Barnes, H. J., J. P. Vaillancourt \& W. B. Gross, 2003. Colibacillosis. In: Diseases of Poultry, $11^{\text {th }}$ edn, Ames: Iowa State University Press, USA, pp. 631-652

Bauer, A. W., W. M. Kirby, J. C. Sherris \& M. Turck, 1966. Antibiotic susceptibility testing by a standardized single disk method. American Journal of Clinical Pathology, 45, 493-496.

Berkhoff, H. A. \& A. C. Vinal, 1986. Congo red medium to distinguish between invasive and non invasive Escherichia coli pathogenic for poultry. Avian Diseases, 30, 117-121.

Cavalieri, S. T., G. A. Bohach \& I. S. Snyder, 1984. E.coli alpha-hemolysis: Characteristics and probable role in pathogenicity. Microbiological Reviews, 48, 326-343.

Gregova, G., M. Kmetova, V. Kmet, J. Venglovsky \& A. Feher, 2012. Antibiotic resistance of Escherichia coli isolated from a poultry slaughterhouse. Annals of Agricultural and Environmental Medicine, 19, 75-77.

Gross, W. B., 1994. Diseases due to Escherichia coli in poultry. In: Escherichia coli in Domestic Animals and Humans, ed. C. L. Gyles, CAB International, Library Wallingford, United Kingdom, pp. 237260.

Guerra, B., E. Junker, A. Schroeter, B. Malorny, S. Lehmann \& R. Helmuth, 2003. Phenotypic and genotypic characterization of antimicrobial resistance in German Escherichia coli isolates from cattle, swine and poultry. Journal of Antimicrobial Chemotherapy, 52, 489-492.

Gundogan, N., A. Devren \& S. Ctak, 2006. Incidence, protease activity and antibiotic resistance of Escherichia coli and Serratia marcescens isolated from meat, chicken and meatball samples. Archiv für Lebensmittelhygiene, 57, 113-117.
Hanson, R., J. B. Kaneene, P. Padungtod, K. Hirokawa \& C. Zeno, 2002. Prevalence of Salmonella and E. coli, and their resistance to antimicrobial agents in farming communities in northern Thailand. Southeast Asian Journal of Tropical Medicine and Public Health, 33, 120-126.

Hawkey, P. M., 2008. Molecular epidemiology of clinically significant antibiotic resistance genes. British Journal of Pharmacology, 153 (Suppl 1), S406-S413.

Johnson, J. R., M. R. Sannes, C. Croy, B. Johnston, C. Clabots, M. A. Kuskowski, J. Bender, K. E. Smith, P. L. Winokur \& E. A. Belongia, 2007. Antimicrobial drugresistant Escherichia coli from humans and poultry products, Minnesota and Wisconsin, 2002-2004. Emerging Infectious Diseases, 13, 838-846.

Khan, A., S. C. Das, T. Ramamurthy, A. Sikdar, J. Khanam, S. Yamasaki, Y. Takeda \& G. B. Nair, 2002. Antibiotic resistance, virulence gene, and molecular profile of shiga toxin-producing Escherichia coli isolates from diverse source in Calcutta, India. Journal of Clinical Microbiology, 40, 2009-2015.

Khoshkhoo, P. H. \& S. M. Peighambari, 2005. Drug resistance patterns and plasmid profiles of Escherichia coli isolated from cases of avian colibacillosis. Journal of Faculty of Veterinary Medicine University of Tehran, 60, 97-105.

Li, L., Z. G. Jiang, L. N. Xia, J. Z. Shen, L. Dai, Y. Wang, S. Y. Huang \& C. M. Wu, 2010. Characterization of antimicrobial resistance and molecular determinants of beta-lactamase in Escherichia coli isolated from chickens in China during 1970-2007. Veterinary Microbiology, 144, 505-510.

Nakamura, K., J. K. A. Cook, J. A. Frazier \& M. Narita, 1992. Escherichia coli multiplication and lesions in the respiratory tract of chickens inoculated with infectious bronchitis virus and/or E. coli. Avian Diseases, 36, 881-890.

Pourbakshsh, S. A., M. Boulianne, B. Martineau-Doizé, C. M. Dozois, C. Desautels \& 
J. F. Fairbrother, 1997. Dynamics of Escherichia coli infection in experimentally inoculated chickens. Avian Diseases, 41, 221-233.

Rahman, M., B. M. Rahman \& B. Rahman, 2008. Antibiogram and plasmid profile analysis of isolated Escherichia coli from broiler and layer. Research in Microbiology, 3, 82-90.

Soulsby, L., 2008. The 2008 Garrod lecture: Antimicrobial resistance-animals and the environment. Journal of Antimicrobial Chemotherapy, 62, 229-233.

Wang, Q., X. Ruan, D. Wei, Z. Hu, L. Wu, T. Yu, L. Feng \& L. Wang, 2010. Development of a serogroup-specific multiplex PCR assay to detect a set of Escherichia coli serogroups based on the identification of their O-antigen gene clusters. Molecular and Cellular Probes, 24, 286-290.

Zakeri, A. \& P. Kashefi, 2012. Antimicrobial susceptibilities of avian Escherichia coli isolates in Tabriz, Iran. African Journal of Biotechnology, 11, 4467-4470.

Paper received 13.03.2014; accepted for publication 25.04.2014

\section{Correspondence:}

Saeed Seifi

Amol University of Special

Modern Technologies

$24^{\text {th }}$ Aftab, Emam Khomeini Street, Amol, Iran

Phone: +98 1212271055;

Fax: +98 1212271054;

e-mail: s.seifi@ausmt.ac.ir 